\title{
Measuring Quantum Coherence with Entanglement
}

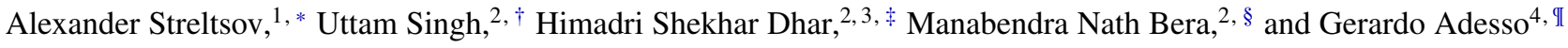 \\ ${ }^{1}$ ICFO-The Institute of Photonic Sciences, Mediterranean Technology Park, 08860 Castelldefels (Barcelona), Spain \\ ${ }^{2}$ Harish-Chandra Research Institute, Chhatnag Road, Jhunsi, Allahabad 211 019, India \\ ${ }^{3}$ School of Physical Sciences, Jawaharlal Nehru University, New Delhi 110 067, India \\ ${ }^{4}$ School of Mathematical Sciences, The University of Nottingham, University Park, Nottingham NG7 2RD, United Kingdom
}

(Dated: June 10, 2015)

\begin{abstract}
Quantum coherence is an essential ingredient in quantum information processing and plays a central role in emergent fields such as nanoscale thermodynamics and quantum biology. However, our understanding and quantitative characterization of coherence as an operational resource are still very limited. Here we show that any degree of coherence with respect to some reference basis can be converted to entanglement via incoherent operations. This finding allows us to define a novel general class of measures of coherence for a quantum system of arbitrary dimension, in terms of the maximum bipartite entanglement that can be generated via incoherent operations applied to the system and an incoherent ancilla. The resulting measures are proven to be valid coherence monotones satisfying all the requirements dictated by the resource theory of quantum coherence. We demonstrate the usefulness of our approach by proving that the fidelity-based geometric measure of coherence is a full convex coherence monotone, and deriving a closed formula for it on arbitrary single-qubit states. Our work provides a clear quantitative and operational connection between coherence and entanglement, two landmark manifestations of quantum theory and both key enablers for quantum technologies.
\end{abstract}

PACS numbers: 03.65.Ud, 03.65.Ta, 03.67.Ac, 03.67.Mn

Introduction.-Coherence is a fundamental aspect of quantum physics that encapsulates the defining features of the theory [1], from the superposition principle to quantum correlations. It is a key component in various quantum information and estimation protocols and is primarily accountable for the advantage offered by quantum tasks versus classical ones $[2,3]$. In general, coherence is an important physical resource in low-temperature thermodynamics [4-8], for exciton and electron transport in biomolecular networks [9-14], and for applications in nanoscale physics $[15,16]$. Experimental detection of coherence in living complexes $[17,18]$ and creation of coherence in hot systems [19] have also been reported.

While the theory of quantum coherence is historically well developed in quantum optics [20-27], a rigorous framework to quantify coherence for general states in information theoretic terms has only been attempted recently [14, 26, 28-31]. This framework is based on identifying the set of incoherent states and a class of 'free' operations, named incoherent quantum channels, that map the set onto itself $[14,28]$. The resulting resource theory of coherence is in direct analogy with the resource theory of entanglement [32], in which local operations and classical communication are the 'free' operations that map the set of separable states onto itself [33]. Within such a framework for coherence, one can define suitable measures that vanish for any incoherent state, and satisfy specific monotonicity requirements under incoherent channels. Measures that respect these conditions gain the attribute of coherence monotones, in analogy with entanglement monotones

\footnotetext{
* alexander.streltsov@icfo.es

† uttamsingh@hri.res.in

\$ himadrisdhar@hri.res.in

§manabbera@hri.res.in

II gerardo.adesso@nottingham.ac.uk
}

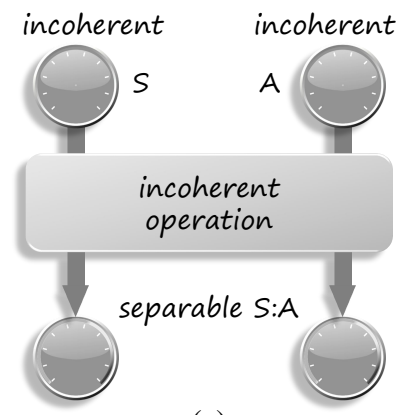

(a)

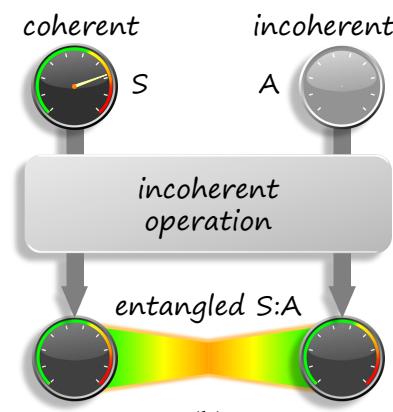

(b)
Figure 1. (a) Incoherent operations cannot generate entanglement from incoherent input states. (b) Conversely, we show that any nonzero coherence in the input state of a system $S$ can be converted to entanglement via incoherent operations on $S$ and an incoherent ancilla $A$. Input coherence and output entanglement are quantitatively equivalent: For any entanglement monotone $E$, the maximum entanglement generated between $S$ and $A$ by incoherent operations defines a faithful coherence monotone $C_{E}$ on the initial state of $S$.

[34]. Examples of coherence monotones include the relative entropy and the $l_{1}$-norm of coherence [28]. Intuitively, both coherence and entanglement capture quantumness of a physical system, and it is well known that entanglement stems from the superposition principle, which is also the essence of coherence. It is then legitimate to ask how can one resource emerge quantitatively from the other [24, 26].

In this Letter, we provide a mathematically rigorous approach to resolve the above question, using a common frame to quantify quantumness in terms of coherence and entanglement. In particular, we show that any nonzero amount of coherence in a system $S$ can be converted to (distillable) entanglement between $S$ and an initially incoherent ancilla $A$, by means of incoherent operations. This allows us to formu- 
late a novel, general method to quantify coherence in terms of entanglement (see Fig. 1). Namely we prove that, given an arbitrary set of entanglement monotones $\{E\}$, one can define a corresponding class of coherence monotones $\left\{C_{E}\right\}$ that satisfy all the requirements of Ref. [28]. The input coherence $C_{E}$ of $S$ is defined as the maximum output entanglement $E$ over all incoherent operations on $S$ and $A$. We explicitly evaluate the maximization in some relevant instances, defining novel coherence monotones such as the fidelity-based geometric measure of coherence. These results provide powerful advances for the operational quantification of coherence.

Characterizing coherence.-For an arbitrary fixed reference basis $\{|i\rangle\}$, the incoherent states are defined as [28]

$$
\sigma=\sum_{i} p_{i}|i\rangle\langle i|
$$

where $p_{i}$ are probabilities. Any state which cannot be written as above is defined coherent [28]. Note that, unlike other resources in information theory, coherence is basis-dependent. The reference basis with respect to which coherence is measured depends on the physical problem under investigation; it is e.g. the energy basis for transport phenomena in engineered and biological domains [13], or the eigenbasis of the generator of an unknown phase shift in quantum metrology [2].

A completely positive trace preserving map $\Lambda$ is said to be an incoherent operation if it can be written as

$$
\Lambda[\rho]=\sum_{l} K_{l} \rho K_{l}^{\dagger},
$$

where the defining operators $K_{l}$, called incoherent Kraus operators, map every incoherent state to some other incoherent state, i.e. $K_{l} \mathcal{I} K_{l}^{\dagger} \subseteq \mathcal{I}$, where $\mathcal{I}$ is the set of incoherent states.

Following established notions from entanglement theory [32, 35-37], Baumgratz et al. proposed the following postulates for a measure of coherence $C(\rho)$ in Ref. [28]:

- (C1) $C(\rho) \geq 0$, and $C(\sigma)=0$ if and only if $\sigma \in \mathcal{I}$.

- (C2) $C(\rho)$ is nonincreasing under incoherent operations, i.e., $C(\rho) \geq C(\Lambda[\rho])$ with $\Lambda[\mathcal{I}] \subseteq \mathcal{I}$.

- (C3) $C(\rho)$ is nonincreasing on average under selective incoherent operations, i.e., $C(\rho) \geq \sum_{l} p_{l} C\left(\varsigma_{l}\right)$, with probabilities $p_{l}=\operatorname{Tr}\left[K_{l} \rho K_{l}^{\dagger}\right]$, states $\varsigma_{l}=K_{l} \rho K_{l}^{\dagger} / p_{l}$, and incoherent Kraus operators $K_{l}$ obeying $K_{l} \mathcal{I} K_{l}^{\dagger} \subseteq \mathcal{I}$.

- (C4) $C(\rho)$ is a convex function of density matrices, i.e., $C\left(\sum_{i} p_{i} \rho_{i}\right) \leq \sum_{i} p_{i} C\left(\rho_{i}\right)$.

Note that conditions (C3) and (C4) automatically imply condition (C2). The reason we listed all conditions above is that (similar to entanglement measures) there might exist meaningful quantifiers of coherence which satisfy conditions (C1) and (C2), but for which conditions (C3) and (C4) are either violated or cannot be proven. Following the analogous notion from entanglement theory, we call a quantity which satisfies conditions $(\mathrm{C} 1),(\mathrm{C} 2)$, and $(\mathrm{C} 3)$ a coherence monotone.

Examples of functionals that satisfy all the four properties mentioned above include the $l_{1}$-norm of coherence [28] $C_{l_{1}}(\rho)=\sum_{i \neq j}\left|\rho_{i j}\right|$ and the relative entropy of coherence [28]

$$
C_{\mathrm{r}}(\rho)=\min _{\sigma \in \mathcal{I}} H(\rho \| \sigma)
$$

with the quantum relative entropy $H\left(\rho \|_{\zeta}\right)=\operatorname{Tr}\left[\rho \log _{2} \rho\right]-$ $\operatorname{Tr}\left[\rho \log _{2} \varsigma\right]$. As was shown in [28], the relative entropy of coherence can also be written as $C_{\mathrm{r}}(\rho)=H\left(\rho_{d}\right)-H(\rho)$, where $\rho_{d}$ is the diagonal part of the density matrix $\rho$ in the reference basis $\{|i\rangle\}$ and $H$ is the von Neumann entropy.

Bipartite coherence.-We first extend the framework of coherence to the bipartite scenario (see also [38]); the following definitions extend straightforwardly to multipartite systems. In particular, for a bipartite system with two subsystems $X$ and $Y$, and with respect to a fixed reference product basis $\left\{|i\rangle^{X} \otimes|j\rangle^{Y}\right\}$, we define bipartite incoherent states as follows:

$$
\rho^{X Y}=\sum_{k} p_{k} \sigma_{k}^{X} \otimes \tau_{k}^{Y}
$$

Here, $p_{k}$ are probabilities and the states $\sigma_{k}^{X}$ and $\tau_{k}^{Y}$ are incoherent states on the subsystem $X$ and $Y$ respectively, i.e. $\sigma_{k}^{X}=$ $\sum_{i} p_{i k}^{\prime}|i\rangle\left\langle\left. i\right|^{X}\right.$ and $\left.\tau_{k}^{Y}=\sum_{j} p_{j k}^{\prime \prime} \mid j\right\rangle\left\langle\left. j\right|^{Y}\right.$ for probabilities $p_{i k}^{\prime}$ and $p_{j k}^{\prime \prime}$. Note that the states in Eq. (4) are always separable.

We next define bipartite incoherent operations as in Eq. (2), with incoherent Kraus operators $K_{l}$ such that $K_{l} \mathcal{I} K_{l}^{\dagger} \subseteq \mathcal{I}$, where $\mathcal{I}$ is now the set of bipartite incoherent states defined in Eq. (4). An example of bipartite incoherent operation is the two-qubit CNOT gate $U_{\mathrm{CNOT}}$. It is not possible to create coherence from an incoherent two-qubit state by using the CNOT gate, since it takes any pure incoherent state $|i\rangle \otimes|j\rangle$ to another pure incoherent state, $U_{\mathrm{CNOT}}(|i\rangle \otimes|j\rangle)=$ $|i\rangle \otimes|\bmod (i+j, 2)\rangle$. The CNOT gate can be used however to create entanglement, e.g. it is well known that the state $U_{\mathrm{CNOT}}(|\psi\rangle \otimes|0\rangle)$ is entangled for any coherent state $|\psi\rangle[3]$.

Converting coherence to entanglement.-Referring to Fig. 1, we say that the coherence in the initial state $\rho^{S}$ of a (finitedimensional) system $S$ can be converted to entanglement via incoherent operations if, by attaching an ancilla $A$ initialized in a reference incoherent state $|0\rangle\left\langle\left. 0\right|^{A}\right.$, the final system-ancilla state $\Lambda^{S A}\left[\rho^{S} \otimes|0\rangle\left\langle\left. 0\right|^{A}\right]\right.$ is entangled for some incoherent operation $\Lambda^{S A}$. Note that incoherent system states $\sigma^{S}$ cannot be used for conversion to entangled states in this way, since for any incoherent state $\sigma^{S}$ the state $\Lambda^{S A}\left[\sigma^{S} \otimes|0\rangle\left\langle\left. 0\right|^{A}\right]\right.$ will be of the form given in Eq. (4), and thus separable.

Entanglement can instead be generated by incoherent operations if the initial $\rho^{S}$ is coherent, as in the two-qubit CNOT example. It is then natural to ask: Can any nonzero amount of coherence be converted to entanglement via incoherent operations? To answer this, we first consider distance-based measures of entanglement $E_{D}$ and coherence $C_{D}$ [28, 35-38]:

$$
E_{D}(\rho)=\min _{\chi \in \mathcal{S}} D(\rho, \chi), \quad C_{D}(\rho)=\min _{\sigma \in \mathcal{I}} D(\rho, \sigma) .
$$

Here, $\mathcal{S}$ is the set of separable states and $\mathcal{I}$ is the set of incoherent states. Moreover, we demand that the distance $D$ be contractive under quantum operations,

$$
D(\Lambda[\rho], \Lambda[\varsigma]) \leq D(\rho, \varsigma)
$$

for any completely positive trace preserving map $\Lambda$. This implies that $E_{D}$ does not increase under local operations and classical communication $[35,36]$, and $C_{D}$ does not increase under incoherent operations [28]. Equipped with these tools we are now in position to present the first result of this Letter. 
Theorem 1. For any contractive distance $D$, the amount of (distance-based) entanglement $E_{D}$ generated from a state $\rho^{S}$ via an incoherent operation $\Lambda^{S A}$ is bounded above by its (distance-based) coherence $C_{D}$ :

$$
E_{D}^{S: A}\left(\Lambda^{S A}\left[\rho^{S} \otimes|0\rangle\left\langle\left. 0\right|^{A}\right]\right) \leq C_{D}\left(\rho^{S}\right) .\right.
$$

Proof. Let $\sigma^{S}$ be the closest incoherent state to $\rho^{S}$, i.e., $C_{D}\left(\rho^{S}\right)=D\left(\rho^{S}, \sigma^{S}\right)$. The contractivity of the distance $D$ further implies the equality: $D\left(\rho^{S}, \sigma^{S}\right)=D\left(\rho^{S} \otimes|0\rangle\left\langle\left. 0\right|^{A}, \sigma^{S} \otimes\right.\right.$ $|0\rangle\left\langle\left. 0\right|^{A}\right)$. In the final step, note that the application of an incoherent operation $\Lambda^{S A}$ to the incoherent state $\sigma^{S} \otimes|0\rangle\left\langle\left. 0\right|^{A}\right.$ brings it to another incoherent-and thus separable-state. Applying Eq. (6) and combining the aforementioned results we arrive at the desired inequality: $C_{D}\left(\rho^{S}\right) \geq D\left(\Lambda^{S A}\left[\rho^{S} \otimes\right.\right.$ $|0\rangle\left\langle\left. 0\right|^{A}\right], \Lambda^{S A}\left[\sigma^{S} \otimes|0\rangle\left\langle\left. 0\right|^{A}\right]\right) \geq E_{D}^{S: A}\left(\Lambda^{S A}\left[\rho^{S} \otimes|0\rangle\left\langle\left. 0\right|^{A}\right]\right)\right.$.

This result provides a strong link between the resource frameworks of entanglement and coherence. An even stronger link exists when choosing specifically $D$ as the relative entropy. The corresponding quantifiers are the relative entropy of entanglement $E_{\mathrm{r}}$ [35], and the relative entropy of coherence $C_{\mathrm{r}}$ [28] introduced in Eq. (3). Importantly, the inequality (7) can be saturated for these measures if the dimension of the ancilla is not smaller than that of the system, $d_{A} \geq d_{S}$. In this case there always exists an incoherent operation $\Lambda^{S A}$ such that

$$
E_{\mathrm{r}}^{S: A}\left(\Lambda^{S A}\left[\rho^{S} \otimes|0\rangle\left\langle\left. 0\right|^{A}\right]\right)=C_{\mathrm{r}}\left(\rho^{S}\right) .\right.
$$

To prove this statement, we consider the unitary operation

$$
\begin{aligned}
U & =\sum_{i=0}^{d_{S}-1} \sum_{j=0}^{d_{S}-1}|i\rangle\left\langle\left. i\right|^{S} \otimes \mid \bmod \left(i+j, d_{S}\right)\right\rangle\left\langle\left. j\right|^{A}\right. \\
& +\sum_{i=0}^{d_{S}-1} \sum_{j=d_{S}}^{d_{A}-1}|i\rangle\left\langle\left. i\right|^{S} \otimes \mid j\right\rangle\left\langle\left. j\right|^{A} .\right.
\end{aligned}
$$

Note that for two qubits this unitary is equivalent to the CNOT gate with $S$ as the control qubit and $A$ as the target qubit. It can be seen by inspection that this unitary is incoherent (i.e., the state $\Lambda^{S A}\left[\rho^{S A}\right]=U \rho^{S A} U^{\dagger}$ is incoherent for any incoherent state $\left.\rho^{S A}\right)$, and maps the state $\rho^{S} \otimes|0\rangle\left\langle\left. 0\right|^{A}\right.$ to the state

$$
\Lambda^{S A}\left[\rho^{S} \otimes|0\rangle\left\langle\left. 0\right|^{A}\right]=\sum_{i, j} \rho_{i j}|i\rangle\left\langle\left. j\right|^{S} \otimes \mid i\right\rangle\left\langle\left. j\right|^{A},\right.\right.
$$

where $\rho_{i j}$ are the matrix elements of $\rho^{S}=\sum_{i, j} \rho_{i j}|i\rangle\left\langle\left. j\right|^{S}\right.$. In the next step we use the fact that for any quantum state $\varsigma^{S A}$ the relative entropy of entanglement is bounded below as follows [39]: $E_{\mathrm{r}}^{S: A}\left(\varsigma^{S A}\right) \geq H\left(\varsigma^{S}\right)-H\left(\varsigma^{S A}\right)$. Applied to the state $\Lambda^{S A}\left[\rho^{S} \otimes|0\rangle\left\langle\left. 0\right|^{A}\right]\right.$, this inequality reduces to

$$
E_{\mathrm{r}}^{S: A}\left(\Lambda^{S A}\left[\rho^{S} \otimes|0\rangle\left\langle\left. 0\right|^{A}\right]\right) \geq H\left(\sum_{i} \rho_{i i}|i\rangle\left\langle\left. i\right|^{S}\right)-H\left(\rho^{S}\right) .\right.\right.
$$

Noting that the right-hand side of this inequality is equal to the relative entropy of coherence $C_{\mathrm{r}}\left(\rho^{S}\right)$ [28], we obtain $E_{\mathrm{r}}^{S: A}\left(\Lambda^{S A}\left[\rho^{S} \otimes|0\rangle\left\langle\left. 0\right|^{A}\right]\right) \geq C_{\mathrm{r}}\left(\rho^{S}\right)\right.$. The proof of Eq. (8) is complete by combining this result with Theorem 1 .

The results presented above also hold for the distillable entanglement $E_{\mathrm{d}}$. Namely, the relative entropy of coherence $C_{\mathrm{r}}$ also serves as an upper bound for the conversion to distillable entanglement via incoherent operations, and the equality in Eq. (8) still holds if $E_{\mathrm{r}}$ is replaced by $E_{\mathrm{d}}$, and the incoherent unitary of Eq. (9) is applied. This follows from Theorem 1, together with the fact that distillable entanglement admits the following bounds [40, 41]: $H\left(\varsigma^{S}\right)-H\left(\varsigma^{S A}\right) \leq E_{\mathrm{d}}^{S: A} \leq E_{\mathrm{r}}^{S: A}$.

This shows that the degree of (relative entropy of) coherence in the initial state of $S$ can be exactly converted to an equal degree of (distillable or relative entropy of) entanglement between $S$ and the incoherent ancilla $A$ by a suitable incoherent operation, that is a generalized CNOT gate. We can now settle the general question posed above.

Theorem 2. A state $\rho^{S}$ can be converted to an entangled state via incoherent operations if and only if $\rho^{S}$ is coherent.

Proof. If $\rho^{S}$ is incoherent, it cannot be converted to an entangled state via incoherent operations by Theorem 1. Conversely, if $\rho^{S}$ is coherent, it has nonzero relative entropy of coherence $C_{\mathrm{r}}\left(\rho^{S}\right)>0$. By Eq. (8), there exists an incoherent operation $\Lambda^{S A}$ leading to nonzero relative entropy of entanglement $E_{\mathrm{r}}^{S: A}\left(\Lambda^{S A}\left[\rho^{S} \otimes|0\rangle\left\langle\left. 0\right|^{A}\right]\right)>0\right.$, concluding the proof.

Quantifying coherence with entanglement.-We are ready to present the central result of the Letter. Reversing the perspective, Theorem 1 can also be seen as providing a lower bound on distance-based measures of coherence through conversion to entanglement: precisely, the coherence degree $C_{D}$ of a state $\rho^{S}$ is always bounded below by the maximal entanglement degree $E_{D}$ generated from it by incoherent operations.

Going now beyond the specific setting of distance-based measures, we will show that such a maximization of the output entanglement, for any fully general entanglement monotone, leads to a quantity which yields a valid quantifier of input coherence in its own right. We specifically define the family of entanglement-based coherence measures $\left\{C_{E}\right\}$ as follows:

$$
C_{E}\left(\rho^{S}\right)=\lim _{d_{A} \rightarrow \infty}\left\{\sup _{\Lambda^{S A}} E^{S: A}\left(\Lambda^{S A}\left[\rho^{S} \otimes|0\rangle\left\langle\left. 0\right|^{A}\right]\right)\right\} .\right.
$$

Here, $E$ is an arbitrary entanglement measure and the supremum is taken over all incoherent operations $\Lambda^{S A}$ [42].

It is crucial to benchmark the validity of $C_{E}$ for any $E$ as a proper measure of coherence. Remarkably, we find that $C_{E}$ satisfies all the aforementioned conditions $(\mathrm{C} 1)-(\mathrm{C} 3)$ given an arbitrary entanglement monotone $E$, with the addition of (C4) if $E$ is convex as well. We namely get the following result:

Theorem 3. $C_{E}$ is a (convex) coherence monotone for any (convex) entanglement monotone $E$.

Proof. Using the arguments presented above it is easy to see that $C_{E}$ is nonnegative, and zero if and only if the state $\rho^{S}$ is incoherent. Moreover, $C_{E}$ does not increase under incoherent operations $\Lambda^{S}$ performed on the system $S$. This can be seen directly from the definition of $C_{E}$ in Eq. (12), noting that an incoherent operation $\Lambda^{S}$ on the system $S$ is also incoherent with respect to $S A$. The proof that $C_{E}$ further satisfies condition (C3) is presented in the Supplemental Material [43]. There we also show that $C_{E}$ is convex for any convex entanglement monotone $E$, i.e. (C4) is fulfilled as well in this case. 
These powerful results complete the parallel between coherence and entanglement, de facto establishing their full quantitative equivalence within the respective resource theories. Thanks to Theorem 3, one can now use the comprehensive knowledge acquired in entanglement theory in the last two decades [34, 35, 37, 44], to address the quantification of coherence in a variety of operational settings, and to define and validate physically motivated coherence monotones. For instance, $C_{E}$ as defined by Eq. (12) amounts to the previously defined relative entropy of coherence [28], if $E$ is the relative entropy of entanglement or the distillable entanglement.

Furthermore, we can now focus on the relevant case of $E$ being the geometric entanglement $[45,46] E_{\mathrm{g}}$, defined for a bipartite state $\rho$ as $E_{\mathrm{g}}(\rho)=1-\max _{\chi \in \mathcal{S}} F(\rho, \chi)$, where the maximum is taken over all separable states $\chi \in \mathcal{S}$, and $F(\rho, \varsigma)=$ $\left(\operatorname{Tr}(\sqrt{\rho} \varsigma \sqrt{\rho})^{1 / 2}\right)^{2}$ is the Uhlmann fidelity. The geometric entanglement coincides with its expression obtained via convex roof $[46,47], E_{\mathrm{g}}(\rho)=\min \sum_{i} p_{i} E_{\mathrm{g}}\left(\left|\psi_{i}\right\rangle\right)$, where the minimum is over all decompositions of $\rho=\sum_{i} p_{i}\left|\psi_{i}\right\rangle\left\langle\psi_{i}\right|$. In the Supplemental Material [43], we show that the geometric measure of coherence $C_{\mathrm{g}}$, associated to $E_{\mathrm{g}}$ via Eq. (12), can be evaluated explicitly and amounts to $C_{\mathrm{g}}(\rho)=1-\max _{\sigma \in \mathcal{I}} F(\rho, \sigma)$, where the maximum is taken over all incoherent states $\sigma \in \mathcal{I}$. The incoherent operation which attains the maximum in Eq. (12) is again the generalized CNOT defined by Eq. (9). Due to Theorem 3, since the geometric measure of entanglement is a full convex entanglement monotone [37, 45], we have just proven that the geometric measure of coherence $C_{\mathrm{g}}$ is a full convex coherence monotone obeying (C1)-(C4). This settles an important question left open in previous literature [28, 38]. Remarkably, the geometric measure $C_{\mathrm{g}}$ is also analytically computable for an arbitrary state $\rho$ of one qubit [43], as follows:

$$
C_{\mathrm{g}}(\rho)=\frac{1}{2}\left(1-\sqrt{1-4\left|\rho_{01}\right|^{2}}\right)
$$

where $\rho_{01}$ is the off-diagonal element of $\rho$ with respect to the reference basis. Notice that $C_{\mathrm{g}}$ in this case is a simple monotonic function of the $l_{1}$-norm of coherence [28], $C_{l_{1}}(\rho)=2\left|\rho_{01}\right|$.

Some of these results extend to any distance-based entanglement measure $E_{g(F)}$ defined via Eq. (5) with $D_{g(F)}(\rho, \varsigma)=$ $g(F(\rho, \varsigma))$, where $g(F)$ is a nonincreasing function of the fidelity $F$. These include the Bures measure of entanglement $[35,36]$, with $g(F)=2(1-\sqrt{F})$, and the Groverian measure of entanglement [48, 49], with $g(F)=\sqrt{1-F}$. For any such entanglement $E_{g(F)}$, the corresponding quantifier of coherence is $C_{g(F)}(\rho)=\min _{\sigma \in \mathcal{I}} D_{g(F)}(\rho, \sigma)$ [43], and Theorem 1 holds with equality for any matching pair $E_{g(F)}$ and $C_{g(F)}$ [50]. Conclusions.- In this Letter we have established a rigorous and general framework for the interconversion between two quantum resources, coherence on one hand, and entanglement on the other hand, via incoherent operations. Our framework can be interpreted in both ways: on one hand, it demonstrates the formal potential of coherence for entanglement generation (although not necessarily useful in practical applications, as cheaper schemes for entanglement creation might be available); on the other hand, it demonstrates the usefulness of entanglement to obtain and validate measures of coherence.
Building on this connection, we proposed in fact a family of coherence quantifiers in terms of the maximal entanglement that can be generated by incoherent operations (see Fig. 1). The proposed coherence quantifiers satisfy all the necessary criteria to be bona fide coherence monotones [28]. In particular, the relative entropy of coherence and the geometric measure of coherence have been (re)defined and interpreted operationally in terms of the maximum converted distillable and geometric entanglement, respectively.

Our framework bears some resemblance with, and may be regarded as the general finite-dimensional counterpart to, the established (qualitative and quantitative) equivalence between input nonclassicality, intended as superposition of optical coherent states, and output entanglement created by passive quantum optical elements such as beam splitters [23, 24, $26,61]$. The results presented here should also be compared to the scheme for activating distillable entanglement via premeasurement interactions [51-53] from quantum discord, a measure of nonclassical correlations going beyond entanglement $[54,55]$. In the latter approach, which has attracted a large amount of attention recently [54, 56-59], measures of discord in a composite system are defined in terms of the minimum entanglement created with an ancillary system via fixed premeasurement interactions defined as in Eq. (9), where the minimization is over local unitaries on the system regulating the control bases before the interaction. By contrast, in this work the reference basis is fixed, and a maximization of the output entanglement over all incoherent operations returns a measure of coherence for the initial system. One might combine the two approaches in order to define a unified framework for interconversion among coherence, discord, and entanglement, whereby discord-type measures could be interpreted as measures of bipartite coherence suitably minimized over local product reference bases (see e.g. [38, 62, 63]). Exploring these connections further will be the subject of another work.

The theory of entanglement has been the cornerstone of major developments in quantum information theory and has triggered the advancement of modern quantum technologies. The construction of a physically meaningful and mathematically rigorous quantitative theory of coherence can improve our perception of genuine quantumness, and guide our understanding of nascent fields such as quantum biology and nanoscale thermodynamics. By uncovering a powerful operational connection between coherence and entanglement, we believe the present work delivers a substantial step in this direction.

Acknowledgments.-We thank M. Ahmadi, J. Asboth, R. Augusiak, T. Bromley, M. Cianciaruso, W. Vogel, A. Winter, and especially M. Piani for fruitful discussions. A.S. acknowledges financial support by the Alexander von HumboldtFoundation, the John Templeton Foundation, the EU (IP SIQS), the ERC AdG OSYRIS, and the EU-Spanish Ministry (CHISTERA DIQIP). U.S., H.S.D., and M.N.B. acknowledge the Department of Atomic Energy, Government of India for research fellowship. G.A. acknowledges financial support by the Foundational Questions Institute (Grant No. FQXiRFP3-1317) and the ERC StG GQCOP (Grant Agreement No. 637352). 
[1] A. J. Leggett, Prog. Theor. Phys. Suppl. 69, 80 (1980).

[2] V. Giovannetti, S. Lloyd, and L. Maccone, Nat. Photon. 5, 222 (2011).

[3] M. A. Nielsen and I. L. Chuang, Quantum Computation and Quantum Information (Cambridge University Press, 2010).

[4] J. Åberg, Phys. Rev. Lett. 113, 150402 (2014).

[5] V. Narasimhachar and G. Gour, arXiv:1409.7740.

[6] P. Ćwikliński, M. Studziński, M. Horodecki, and J. Oppenheim, arXiv:1405.5029.

[7] M. Lostaglio, D. Jennings, and T. Rudolph, Nat. Commun. 6, 6383 (2015).

[8] M. Lostaglio, K. Korzekwa, D. Jennings, and T. Rudolph, Phys. Rev. X 5, 021001 (2015).

[9] M. B. Plenio and S. F. Huelga, New J. Phys. 10, 113019 (2008).

[10] P. Rebentrost, M. Mohseni, and A. Aspuru-Guzik, J. Phys. Chem. B 113, 9942 (2009).

[11] S. Lloyd, J. Phys.: Conf. Ser. 302, 012037 (2011).

[12] C.-M. Li, N. Lambert, Y.-N. Chen, G.-Y. Chen, and F. Nori, Sci. Rep. 2, 885 (2012).

[13] S. Huelga and M. Plenio, Contemporary Physics 54, 181 (2013).

[14] F. Levi and F. Mintert, New J. Phys. 16, 033007 (2014).

[15] H. Vazquez, R. Skouta, S. Schneebeli, M. Kamenetska, R. Breslow, L. Venkataraman, and M. Hybertsen, Nat. Nanotech. 7, 663 (2012).

[16] O. Karlström, H. Linke, G. Karlström, and A. Wacker, Phys. Rev. B 84, 113415 (2011).

[17] G. S. Engel et al., Nature 446, 782 (2007).

[18] E. Collini et al., Nature 463, 644 (2010).

[19] L. Rybak, S. Amaran, L. Levin, M. Tomza, R. Moszynski, R. Kosloff, C. P. Koch, and Z. Amitay, Phys. Rev. Lett. 107, 273001 (2011).

[20] R. J. Glauber, Phys. Rev. 131, 2766 (1963).

[21] E. C. G. Sudarshan, Phys. Rev. Lett. 10, 277 (1963).

[22] L. Mandel and E. Wolf, Optical Coherence and Quantum Optics (Cambridge University Press, 1995).

[23] M. S. Kim, W. Son, V. Bužek, and P. L. Knight, Phys. Rev. A 65, 032323 (2002).

[24] J. K. Asbóth, J. Calsamiglia, and H. Ritsch, Phys. Rev. Lett. 94, 173602 (2005).

[25] T. Richter and W. Vogel, Phys. Rev. Lett. 89, 283601 (2002).

[26] W. Vogel and J. Sperling, Phys. Rev. A 89, 052302 (2014).

[27] M. Mraz, J. Sperling, W. Vogel, and B. Hage, Phys. Rev. A 90, 033812 (2014).

[28] T. Baumgratz, M. Cramer, and M. B. Plenio, Phys. Rev. Lett. 113, 140401 (2014).

[29] D. Girolami, Phys. Rev. Lett. 113, 170401 (2014).

[30] C. Smyth and G. D. Scholes, Phys. Rev. A 90, 032312 (2014).

[31] D. P. Pires, L. C. Celeri, and D. O. Soares-Pinto, arXiv:1501.05271.

[32] R. Horodecki, P. Horodecki, M. Horodecki, and K. Horodecki, Rev. Mod. Phys. 81, 865 (2009).

[33] F. G. S. L. Brandão, M. Horodecki, J. Oppenheim, J. M. Renes, and R. W. Spekkens, Phys. Rev. Lett. 111, 250404 (2013).

[34] G. Vidal, J. Mod. Opt. 47, 355 (2000).

[35] V. Vedral, M. B. Plenio, M. A. Rippin, and P. L. Knight, Phys. Rev. Lett. 78, 2275 (1997).

[36] V. Vedral and M. B. Plenio, Phys. Rev. A 57, 1619 (1998).

[37] M. B. Plenio and S. Virmani, Quantum Inf. Comput. 7, 1 (2007).

[38] T. R. Bromley, M. Cianciaruso, and G. Adesso, Phys. Rev. Lett.
114, 210401 (2015).

[39] M. B. Plenio, S. Virmani, and P. Papadopoulos, J. Phys. A 33, L193 (2000)

[40] M. Horodecki, P. Horodecki, and R. Horodecki, Phys. Rev. Lett. 84, 2014 (2000).

[41] I. Devetak and A. Winter, Proc. R. Soc. A 461, 207 (2005).

[42] Note that the limit $d_{A} \rightarrow \infty$ in Eq. (12) is well defined, since the supremum $\sup _{\Lambda^{S A}} E^{S: A}\left(\Lambda^{S A}\left[\rho^{S} \otimes|0\rangle\left\langle\left. 0\right|^{A}\right]\right)\right.$ cannot decrease with increasing dimension $d_{A}$ of the ancilla.

[43] See the Supplemental Material for technical derivations.

[44] W. K. Wootters, Phys. Rev. Lett. 80, 2245 (1998).

[45] T.-C. Wei and P. M. Goldbart, Phys. Rev. A 68, 042307 (2003).

[46] A. Streltsov, H. Kampermann, and D. Bruß, New J. Phys. 12, 123004 (2010).

[47] M. Horodecki, A. Sen(De), and U. Sen, Phys. Rev. A 67, 062314 (2003).

[48] O. Biham, M. A. Nielsen, and T. J. Osborne, Phys. Rev. A 65, 062312 (2002).

[49] D. Shapira, Y. Shimoni, and O. Biham, Phys. Rev. A 73, 044301 (2006).

[50] This defines a whole new class of operational coherence measures. Note, however, that not all these measures will satisfy properties (C3) or (C4) [60], as the corresponding entanglement measures are not all monotonic under selective LOCC operations or convex; this depends on the specific form of $g(F)$.

[51] M. Piani, S. Gharibian, G. Adesso, J. Calsamiglia, P. Horodecki, and A. Winter, Phys. Rev. Lett. 106, 220403 (2011).

[52] A. Streltsov, H. Kampermann, and D. Bruß, Phys. Rev. Lett. 106, 160401 (2011).

[53] S. Gharibian, M. Piani, G. Adesso, J. Calsamiglia, and P. Horodecki, Int. J. Quantum. Inform. 09, 1701 (2011).

[54] K. Modi, A. Brodutch, H. Cable, T. Paterek, and V. Vedral, Rev. Mod. Phys. 84, 1655 (2012).

[55] A. Streltsov, Quantum Correlations Beyond Entanglement and their Role in Quantum Information Theory (SpringerBriefs in Physics, 2015) arXiv:1411.3208.

[56] M. Piani and G. Adesso, Phys. Rev. A 85, 040301 (2012).

[57] T. Nakano, M. Piani, and G. Adesso, Phys. Rev. A 88, 012117 (2013).

[58] G. Adesso, V. D’Ambrosio, E. Nagali, M. Piani, and F. Sciarrino, Phys. Rev. Lett. 112, 140501 (2014).

[59] A. Orieux, M. A. Ciampini, P. Mataloni, D. Bruß, M. Rossi, and C. Macchiavello, arXiv:1503.05084.

[60] L.-H. Shao, Z. Xi, H. Fan, and Y. Li, Phys. Rev. A 91, 042120 (2015).

[61] N. Killoran, F. E. S. Steinhoff, and M. B. Plenio, arXiv:1505.07393.

[62] G. Adesso, Coherence, Discord, and Entanglement: Activating one resource into another and beyond, talk presented at the 586 WE-Heraeus-Seminar on "Quantum Correlations Beyond Entanglement” (Bad Honnef, April 2015).

[63] Y. Yao, X. Xiao, L. Ge, and C. P. Sun, arXiv:1506.01773. 


\section{Appendix: Supplemental Material} Measuring Quantum Coherence with Entanglement

\section{Proof of monotonicity (C3) in Theorem 3}

Here we prove that for any entanglement monotone $E$ the coherence quantifier

$$
C_{E}\left(\rho^{S}\right)=\lim _{d_{A} \rightarrow \infty}\left\{\sup _{\Lambda^{S A}} E^{S: A}\left(\Lambda^{S A}\left[\rho^{S} \otimes|0\rangle\left\langle\left. 0\right|^{A}\right]\right)\right\}\right.
$$

does not increase on average under (selective) incoherent operations:

$$
\sum_{i} p_{i} C_{E}\left(\sigma_{i}^{S}\right) \leq C_{E}\left(\rho^{S}\right)
$$

with probabilities $p_{i}=\operatorname{Tr}\left[K_{i} \rho^{S} K_{i}^{\dagger}\right]$, quantum states $\sigma_{i}^{S}=$ $K_{i} \rho^{S} K_{i}^{\dagger} / p_{i}$, and incoherent Kraus operators $K_{i}$ acting on the system $S$.

Due to the definition of $C_{E}$, the amount of entanglement between the system and ancilla cannot exceed $C_{E}$ for any incoherent operation $\Lambda^{S A}$, i.e.,

$$
E^{S: A}\left(\Lambda^{S A}\left[\rho^{S} \otimes|0\rangle\left\langle\left. 0\right|^{A}\right]\right) \leq C_{E}\left(\rho^{S}\right) .\right.
$$

Note that this statement is also true if we introduce another particle $B$ in an incoherent state $|0\rangle\left\langle\left. 0\right|^{B}\right.$. Then, for any tripartite incoherent operation $\Lambda^{S A B}$ it holds:

$$
E^{S: A B}\left(\Lambda^{S A B}\left[\rho^{S} \otimes|0\rangle\left\langle\left. 0\right|^{A} \otimes \mid 0\right\rangle\left\langle\left. 0\right|^{B}\right]\right) \leq C_{E}\left(\rho^{S}\right) .\right.
$$

We will now prove the claim by contradiction, showing that a violation of Eq. (A.2) also implies a violation of Eq. (A.4). If Eq. (A.2) is violated, then by definition of $C_{E}$ there exists a set of incoherent operations $\Lambda_{i}^{S A}$ such that the following inequality is true for $d_{A}$ large enough:

$$
\sum_{i} p_{i} E^{S: A}\left(\Lambda_{i}^{S A}\left[\sigma_{i}^{S} \otimes|0\rangle\left\langle\left. 0\right|^{A}\right]\right)>C_{E}\left(\rho^{S}\right) .\right.
$$

In the next step we introduce an additional particle $B$ and use the general relation

$$
E^{S: A B}\left(\sum_{i} p_{i} \rho_{i}^{S A} \otimes|i\rangle\left\langle\left. i\right|^{B}\right) \geq \sum_{i} p_{i} E^{S: A}\left(\rho_{i}^{S A}\right)\right.
$$

which is valid for any entanglement monotone $E$. With this in mind, the inequality (A.5) implies

$$
E^{S: A B}\left(\sum _ { i } p _ { i } \Lambda _ { i } ^ { S A } \left[\sigma_{i}^{S} \otimes|0\rangle\left\langle\left. 0\right|^{A}\right] \otimes|i\rangle\left\langle\left. i\right|^{B}\right)>C_{E}\left(\rho^{S}\right) .\right.\right.
$$

Recall that the states $\sigma_{i}^{S}$ are obtained from the state $\rho^{S}$ by the means of an incoherent operation, and thus we can use the relation $p_{i} \sigma_{i}^{S}=K_{i} \rho^{S} K_{i}^{\dagger}$ with incoherent Kraus operators $K_{i}$. This leads us to the following expression:

$$
E^{S: A B}\left(\sum _ { i } \Lambda _ { i } ^ { S A } \left[K_{i} \rho^{S} K_{i}^{\dagger} \otimes|0\rangle\left\langle\left. 0\right|^{A}\right] \otimes|i\rangle\left\langle\left. i\right|^{B}\right)>C_{E}\left(\rho^{S}\right)\right.\right.
$$

It is now crucial to note that the state on the left-hand side of the above expression can be regarded as arising from a tripartite incoherent operation $\Lambda^{S A B}$ acting on the total state $\rho^{S} \otimes|0\rangle\left\langle\left. 0\right|^{A} \otimes \mid 0\right\rangle\left\langle\left. 0\right|^{B}\right.$ :

$$
\begin{aligned}
& \Lambda^{S A B}\left[\rho^{S} \otimes|0\rangle\left\langle\left. 0\right|^{A} \otimes \mid 0\right\rangle\left\langle\left. 0\right|^{B}\right]\right. \\
& =\sum_{i} \Lambda_{i}^{S A}\left[K _ { i } \rho ^ { S } K _ { i } ^ { \dagger } \otimes | 0 \rangle \langle 0 | ^ { A } ] \otimes | i \rangle \left\langle\left.i\right|^{B} .\right.\right.
\end{aligned}
$$

This can be seen explicitly by introducing the Kraus operators $M_{i j}$ corresponding to the operation $\Lambda^{S A B}$ :

$$
M_{i j}^{S A B}=L_{i j}^{S A}\left(K_{i}^{S} \otimes \mathbb{1}^{A}\right) \otimes U_{i}^{B} .
$$

Here, $L_{i j}$ are incoherent Kraus operators corresponding to the incoherent operation $\Lambda_{i}^{S A}$ :

$$
\Lambda_{i}^{S A}\left[\rho^{S A}\right]=\sum_{j} L_{i j} \rho^{S A} L_{i j}^{\dagger}
$$

The unitaries $U_{i}^{B}$ are incoherent and defined as

$$
U_{i}^{B}=\sum_{j=0}^{d_{B}-1}\left|\bmod \left(i+j, d_{B}\right)\right\rangle\left\langle\left. j\right|^{B} .\right.
$$

With these definitions we see that $M_{i j}$ are indeed incoherent Kraus operators. Moreover, it can be verified by inspection that the incoherent operation $\Lambda^{S A B}$ arising from these Kraus operators also satisfies Eq. (A.9).

Finally, using Eq. (A.9) in Eq. (A.8) we arrive at the following inequality:

$$
E^{S: A B}\left(\Lambda^{S A B}\left[\rho^{S} \otimes|0\rangle\left\langle\left. 0\right|^{A} \otimes \mid 0\right\rangle\left\langle\left. 0\right|^{B}\right]\right)>C_{E}\left(\rho^{S}\right) .\right.
$$

This is the desired contradiction to Eq. (A.4), and completes the proof of property (C3) for $C_{E}$, thus establishing that $C_{E}$ is a coherence monotone for any entanglement monotone $E$.

\section{Proof of convexity (C4) in Theorem 3}

Here we show that the quantifier of coherence $C_{E}$ given in Eq. (A.1) is convex for any convex entanglement measure $E$ :

$$
C_{E}\left(\sum_{i} p_{i} \rho_{i}^{S}\right) \leq \sum_{i} p_{i} C_{E}\left(\rho_{i}^{S}\right)
$$

for any quantum states $\rho_{i}^{S}$ and probabilities $p_{i}$. For this, note that by convexity of the entanglement quantifier $E$ it follows:

$$
\begin{aligned}
& E^{S: A}\left(\Lambda^{S A}\left[\sum_{i} p_{i} \rho_{i}^{S} \otimes|0\rangle\left\langle\left. 0\right|^{A}\right]\right)\right. \\
& \leq \sum_{i} p_{i} E^{S: A}\left(\Lambda^{S A}\left[\rho_{i}^{S} \otimes|0\rangle\left\langle\left. 0\right|^{A}\right]\right) .\right.
\end{aligned}
$$

Taking the supremum over all incoherent operations $\Lambda^{S A}$ together with the limit $d_{A} \rightarrow \infty$ on both sides of this inequality 
we obtain the following result:

$$
C_{E}\left(\sum_{i} p_{i} \rho_{i}^{S}\right) \leq \lim _{d_{A} \rightarrow \infty} \sup _{\Lambda^{S A}}\left\{\sum_{i} p_{i} E^{S: A}\left(\Lambda^{S A}\left[\rho_{i}^{S} \otimes|0\rangle\left\langle\left. 0\right|^{A}\right]\right)\right\}\right.
$$

Finally, note that the right-hand side of this inequality cannot decrease if the supremum over incoherent operations $\Lambda^{S A}$ and the limit $d_{A} \rightarrow \infty$ are performed on each term of the sum individually:

$$
\begin{aligned}
& \lim _{d_{A} \rightarrow \infty} \sup _{\Lambda^{S A}}\left\{\sum_{i} p_{i} E^{S: A}\left(\Lambda^{S A}\left[\rho_{i}^{S} \otimes|0\rangle\left\langle\left. 0\right|^{A}\right]\right)\right\}\right. \\
& \leq \sum_{i} p_{i} \lim _{d_{A} \rightarrow \infty} \sup _{\Lambda^{S A}} E^{S: A}\left(\Lambda^{S A}\left[\rho_{i}^{S} \otimes|0\rangle\left\langle\left. 0\right|^{A}\right]\right)\right. \\
& =\sum_{i} p_{i} C_{E}\left(\rho_{i}^{S}\right) .
\end{aligned}
$$

Together with Eq. (A.16), this completes the proof of convexity in Eq. (A.14).

\section{Geometric entanglement and coherence}

We will now show that the bound provided in Theorem 1 of the main text can be saturated for the geometric entanglement $E_{\mathrm{g}}$ and geometric coherence $C_{\mathrm{g}}$. The former is defined as

$$
E_{\mathrm{g}}(\rho)=1-\max _{\chi \in \mathcal{S}} F(\rho, \chi),
$$

where the maximum is taken over all separable states $\mathcal{S}$, and

$$
F(\rho, \varsigma)=(\operatorname{Tr} \sqrt{\sqrt{\rho} \varsigma \sqrt{\rho}})^{2}
$$

is the fidelity. The geometric entanglement defined in Eq. (A.18) coincides with its expression obtained via a convex roof construction [46]:

$$
E_{\mathrm{g}}(\rho)=\min \sum_{i} p_{i} E_{\mathrm{g}}\left(\left|\psi_{i}\right\rangle\right)
$$

where the minimum is taken over all decompositions of the state $\rho=\sum_{i} p_{i}\left|\psi_{i}\right\rangle\left\langle\psi_{i}\right|$. The latter expression (A.20) is the original definition of the geometric entanglement for mixed states [45], and the equivalence of Eqs. (A.18) and (A.20) was shown in [46].

The geometric coherence $C_{\mathrm{g}}$ can be defined similarly:

$$
C_{\mathrm{g}}(\rho)=1-\max _{\sigma \in I} F(\rho, \sigma)
$$

where the maximum is taken over all incoherent states $\sigma \in \mathcal{I}$.

Equipped with these tools, we are now in position to prove the saturation of the bound in Theorem 1 for these measures of entanglement and coherence. In particular, we will show the existence of an incoherent operation $\Lambda^{S A}$ such that

$$
E_{\mathrm{g}}^{S: A}\left(\Lambda^{S A}\left[\rho^{S} \otimes|0\rangle\left\langle\left. 0\right|^{A}\right]\right)=C_{\mathrm{g}}\left(\rho^{S}\right)\right.
$$

if the dimension of the ancilla is not smaller than the dimension of the system, $d_{A} \geq d_{S}$. As we will further show, the optimal incoherent operation achieving this equality is the generalized CNOT operation $U$ given in Eq. (9) of the main text.

To prove Eq. (A.22), we first recall that the final state after the application of the generalized CNOT operation $U$ is

$$
\rho_{\mathrm{mc}}^{S A}=U\left(\rho^{S} \otimes|0\rangle\left\langle\left. 0\right|^{A}\right) U^{\dagger}=\sum_{i, j} \rho_{i j}|i\rangle\left\langle\left. j\right|^{S} \otimes \mid i\right\rangle\left\langle\left. j\right|^{A},\right.\right.
$$

where $\rho_{i j}$ are matrix elements of $\rho^{S}=\sum_{i, j} \rho_{i j}|i\rangle\left\langle\left. j\right|^{S}\right.$. States of the form (A.23) are known as maximally correlated states. As shown in section 4 of this Supplemental Material, there always exists a separable maximally correlated state

$$
\chi_{\mathrm{mc}}^{S A}=\sum_{i} q_{i}|i\rangle\left\langle\left. i\right|^{S} \otimes \mid i\right\rangle\left\langle\left. i\right|^{A}\right.
$$

which is a closest separable state to $\rho_{\mathrm{mc}}^{S A}$ :

$$
E_{\mathrm{g}}^{S: A}\left(\rho_{\mathrm{mc}}^{S A}\right)=1-F\left(\rho_{\mathrm{mc}}^{S A}, \chi_{\mathrm{mc}}^{S A}\right)
$$

These results imply that the geometric coherence of $\rho^{S}$ is bounded above by the geometric entanglement of $\rho_{\mathrm{mc}}^{S A}$ :

$$
C_{\mathrm{g}}\left(\rho^{S}\right) \leq E_{\mathrm{g}}^{S: A}\left(\rho_{\mathrm{mc}}^{S A}\right)
$$

This follows by using Eq. (A.25) together with the equality

$$
F\left(\rho_{\mathrm{mc}}^{S A}, \sigma_{\mathrm{mc}}^{S A}\right)=F\left(\rho^{S}, \sigma^{S}\right)
$$

where $\sigma^{S}=\sum_{i} q_{i}|i\rangle\left\langle\left. i\right|^{S}\right.$ is an incoherent state with the same coefficients $q_{i}$ as in Eq. (A.24). On the other hand, Theorem 1 in the main text implies the inequality

$$
C_{\mathrm{g}}\left(\rho^{S}\right) \geq E_{\mathrm{g}}^{S: A}\left(\rho_{\mathrm{mc}}^{S A}\right)
$$

and thus we arrive at the desired statement in Eq. (A.22).

We further note that the arguments presented above can also be applied to any distance-based quantifiers of entanglement $E_{D}$ and coherence $C_{D}$ if the distance is contractive under quantum operations, and for any maximally correlated state $\rho_{\mathrm{mc}}^{S A}$ there exists a separable maximally correlated state $\chi_{\mathrm{mc}}^{S A} \in \mathcal{S}$ (which may depend on $\rho_{\mathrm{mc}}^{S A}$ ) such that

$$
E_{D}^{S: A}\left(\rho_{\mathrm{mc}}^{S A}\right)=D\left(\rho_{\mathrm{mc}}^{S A}, \chi_{\mathrm{mc}}^{S A}\right)
$$

In particular, this is the case for the geometric entanglement and coherence, where the distance is given by $D(\rho, \sigma)=1-$ $F(\rho, \sigma)$. As discussed in the main text, these results can be immediately extended to any distance

$$
D(\rho, \sigma)=g[F(\rho, \sigma)]
$$

which is a nonincreasing function of fidelity. 


\section{Geometric entanglement for maximally correlated states}

In this section we will show that for any maximally correlated state

$$
\rho_{\mathrm{mc}}=\sum_{i, j} \rho_{i j}|i\rangle\langle j|\otimes| i\rangle\langle j|
$$

there exists a separable maximally correlated state

$$
\chi_{\mathrm{mc}}=\sum_{i} q_{i}|i\rangle\langle i|\otimes| i\rangle\langle i|
$$

such that

$$
E_{\mathrm{g}}\left(\rho_{\mathrm{mc}}\right)=1-F\left(\rho_{\mathrm{mc}}, \chi_{\mathrm{mc}}\right)
$$

This can be proven by using results from Refs. [46, 47]. In particular, given a maximally correlated state $\rho_{\mathrm{mc}}$, consider its arbitrary decomposition into pure states $\left|\psi_{k}\right\rangle$ with positive probabilities $p_{k}>0$ such that

$$
\rho_{\mathrm{mc}}=\sum_{k} p_{k}\left|\psi_{k}\right\rangle\left\langle\psi_{k}\right|
$$

As is proven on page 6 in [47], all states $\left|\psi_{k}\right\rangle$ in such a decomposition must be linear combinations of product states $|i\rangle \otimes|i\rangle$ :

$$
\left|\psi_{k}\right\rangle=\sum_{i} c_{i}^{k}|i\rangle \otimes|i\rangle
$$

with complex coefficients $c_{i}^{k}$. Consider now an optimal decomposition of the state $\rho_{\mathrm{mc}}$, i.e., a decomposition which minimizes the average entanglement such that

$$
\sum_{k} p_{k} E_{\mathrm{g}}\left(\left|\psi_{k}\right\rangle\left\langle\psi_{k}\right|\right)=E_{\mathrm{g}}\left(\rho_{\mathrm{mc}}\right) .
$$

We further define product states $\left|\phi_{k}\right\rangle \in \mathcal{S}$ to be the closest product states to $\left|\psi_{k}\right\rangle$ :

$$
E_{\mathrm{g}}\left(\left|\psi_{k}\right\rangle\right)=1-F\left(\left|\psi_{k}\right\rangle,\left|\phi_{k}\right\rangle\right) .
$$

Due to Eq. (A.35), all states $\left|\phi_{k}\right\rangle$ can be chosen as $\left|\phi_{k}\right\rangle=$ $\left|l_{k}\right\rangle \otimes\left|l_{k}\right\rangle$, where the number $l_{k}$ corresponds to the coefficient $c_{l_{k}}^{k}$ of the state $\left|\psi_{k}\right\rangle$ with the maximal absolute value: $\left|c_{l_{k}}^{k}\right|=\max _{i}\left|c_{i}^{k}\right|$.

Finally, consider the separable maximally correlated state

$$
\chi_{\mathrm{mc}}=\sum_{k} q_{k}\left|\phi_{k}\right\rangle\left\langle\phi_{k}\left|=\sum_{k} q_{k}\right| l_{k}\right\rangle\left\langle l_{k}|\otimes| l_{k}\right\rangle\left\langle l_{k}\right|
$$

with probabilities $q_{k}$ defined as

$$
q_{k}=\frac{p_{k}\left[1-E_{\mathrm{g}}\left(\left|\psi_{k}\right\rangle\right)\right]}{1-E_{\mathrm{g}}\left(\rho_{\mathrm{mc}}\right)} .
$$

As we now show, $\chi_{\mathrm{mc}}$ is the desired optimal state, satisfying the equality (A.33). This can be seen by first recalling that the geometric entanglement of the state $\rho_{\mathrm{mc}}$ is bounded above as

$$
E_{\mathrm{g}}\left(\rho_{\mathrm{mc}}\right) \leq 1-F\left(\rho_{\mathrm{mc}}, \chi_{\mathrm{mc}}\right)
$$

since the state $\chi_{\mathrm{mc}}$ is separable. On the other hand, the square root of the fidelity $\sqrt{F}$ satisfies the strong concavity relation

$$
\sqrt{F(\rho, \varsigma)} \geq \sum_{k} \sqrt{p_{k} q_{k} F\left(\left|\psi_{k}\right\rangle,\left|\phi_{k}\right\rangle\right)}
$$

for any two states $\rho=\sum_{k} p_{k}\left|\psi_{k}\right\rangle\left\langle\psi_{k}\right|$ and $\varsigma=\sum_{k} q_{k}\left|\phi_{k}\right\rangle\left\langle\phi_{k}\right|$; see Theorem 9.7 on page 414 in [3] (note that the fidelity defined there is the square root of the fidelity used in our paper). Applied to the maximally correlated states $\rho_{\mathrm{mc}}$ and $\chi_{\mathrm{mc}}$, and using Eqs. (A.37) and (A.39), this inequality becomes

$$
\sqrt{F\left(\rho_{\mathrm{mc}}, \chi_{\mathrm{mc}}\right)} \geq \sqrt{1-E_{\mathrm{g}}\left(\rho_{\mathrm{mc}}\right)}
$$

implying that the geometric entanglement of $\rho_{\mathrm{mc}}$ is bounded below as

$$
E_{\mathrm{g}}\left(\rho_{\mathrm{mc}}\right) \geq 1-F\left(\rho_{\mathrm{mc}}, \chi_{\mathrm{mc}}\right) .
$$

Combining this result with Eq. (A.40) completes the proof of Eq. (A.33).

\section{Geometric coherence for arbitrary single-qubit states}

Earlier we have proven that the optimal incoherent operation which attains the maximization in Eq. (A.1) is the generalized CNOT when $E$ is the geometric measure of entanglement $E_{\mathrm{g}}$. If the system $S$ is a single qubit $\left(d_{S}=2\right)$, the output state of system and ancilla after the CNOT is a twoqubit state. The geometric entanglement $E_{\mathrm{g}}$ of any bipartite two-qubit state $s$ is computable in closed form and given by $[45,46]$

$$
E_{g}^{S: A}(\varsigma)=\frac{1}{2}\left[1-\sqrt{1-C(\varsigma)^{2}}\right],
$$

where $C$ is the concurrence of $\varsigma$ [44].

Let $\rho^{S}=\sum_{i, j=0}^{1} \rho_{i j}|i\rangle\left\langle\left. j\right|^{S}\right.$ be an arbitrary state of the single qubit $S$, written with respect to a reference basis $\{|i\rangle\}$. After applying the CNOT on the above state and the initially incoherent ancilla, we get a maximally correlated two-qubit state

$$
\rho_{\mathrm{mc}}^{S A}=\operatorname{CNOT}\left[\rho^{S} \otimes|0\rangle\left\langle\left. 0\right|^{A}\right]=\sum_{i, j=0}^{1} \rho_{i j}|i\rangle\left\langle\left. j\right|^{S} \otimes \mid i\right\rangle\left\langle\left. j\right|^{A} .\right.\right.
$$

The concurrence of the above maximally correlated state is easily evaluated as [44]

$$
C\left(\rho_{\mathrm{mc}}^{S A}\right)=2\left|\rho_{01}\right| .
$$

The geometric entanglement of $\rho_{\mathrm{mc}}^{S A}$ can be written then as

$$
\begin{aligned}
E_{g}^{S: A}\left(\rho_{\mathrm{mc}}^{S A}\right) & =\frac{1}{2}\left[1-\sqrt{1-C\left(\rho_{\mathrm{mc}}^{S A}\right)^{2}}\right] \\
& =\frac{1}{2}\left[1-\sqrt{1-4\left|\rho_{01}\right|^{2}}\right] .
\end{aligned}
$$

Finally, the geometric coherence for an arbitrary single-qubit state $\rho$ is given by the expression reported in the main text:

$$
C_{g}(\rho)=E_{g}^{S: A}\left(\rho_{\mathrm{mc}}^{S A}\right)=\frac{1}{2}\left[1-\sqrt{1-4\left|\rho_{01}\right|^{2}}\right] .
$$

